\title{
Editorial
}

\section{Zur sozialen Gestalt von Krankheiten}

Zwei übertragbare Krankheiten standen am Beginn und am Ende der diesjährigen Jahrestagung der Schweizerischen Gesellschaft für Sozial- und Präventivmedizin: Tuberkulose und AIDS.

«Tuberkulose bei Schweizern und Ausländern: Präventivmedizinische Implikationen», «Epidemiologische Analyse des Grenzsanitätsdienstes», «Evaluation du dépistage de la tuberculose dans une population d'apprentis et de gymnasiens vaudois» einerseits und «Effektorientierte Evaluation der AIDS-Aufklärung an Zürcher Volksschulen», «Preserver ses chances matrimoniales sans s'exposer au risque du SIDA - le cas des femmes divorcées» anderseits lauteten die Vortragstitel, die die zurückliegende Tagung eröffneten. Thematik des abschliessenden Freitagnachmittags war "AIDS: Stigmatisierung und Diskriminierung von Betroffenen».

Die beiden Krankheiten Tuberkulose und AIDS stehen für zwei unterschiedliche Epochen in der langen Geschichte der Infektionskrankheiten. Die Tuberkulose ist ein Beispiel für eine weitgehend vergangene oder vielleicht auch nur vermeintlich vergangene Epoche, denkt man an den dramatischen Anstieg der Tuberkulosetodesfälle in New York zwischen 1979 und 1986 [1]. Es ist eine Krankheit, in deren Mortalitätsstatistiken sich die soziale Ungleichheit vor Krankheit und Tod deutlich wiederspiegelt. Hauptbetroffene und Opfer dieser Krankheit in New York sind vor allem Obdachlose und Drogenabhängige.

Und anderseits AIDS, die Infektionskrankheit unserer Tage, die die Annahme, für entwickelte, industrialisierte Gesellschaften, sei die Phase der übertragbaren Krankheiten bereits Medizingeschichte, in dramatischer Weise korrigiert hat. Es ist eine Krankheit, die wie keine andere zuvor eine permanente Präsenz in den Medien und in der sozial- und gesellschaftspolitischen Auseinandersetzung erreicht hat.

Krankheiten besitzen immer auch eine gesellschaftliche Dimension, gleichsam eine soziale Gestalt. Tuberkulose und AIDS sind zwei Krankheiten, die in hohem Masse mit spektakulären, wenn auch inhaltlich unterschiedlichen Metaphern befrachtet wurden und werden. Thema dieser Betrachtungsweise ist nicht die Krankheit selbst, sondern die durch eine Krankheit ausgelösten kollektiven Phantasien. In einem brillant geschriebenen Essay («Krankheit als Metapher») beschreibt Susan Sontag Tuberkulose und Krebs als gesellschaftliche Metaphern [2]. Krebs ist hiernach verkürzt gesagt - die Krankheit, die das Böse schlechthin symbolisiert. Weitgehend noch gilt die Gleichsetzung Krebs - Tod, die Alltagssprache bedient sich in dämonisierender Weise der Krebsmetapher. Tuberkulose hingegen, so die Analyse Susan Sontags, ist eine Krankheit, die eine nahezu weihevolle, positive Aura erfahren hat: Eine Krankheit der Übersensiblen, der von Leidenschaft umtriebenen Menschen. Vielleicht am eindrücklichsten wurde dies in Thomas Manns «Der Zauberberg» beschrieben.

Und AIDS? In einer Nachfolgearbeit hat sich Susan Sontag mit den Metaphern beschäftigt, die die Immunschwächekrankheit belasten und verzerren [3]. Diese Krankheit, die so kurz vor dem Ende des zweiten Jahrtausends aufgetreten ist, vermochte apokalyptische Ängste und Katastrophenphantasien auszulösen. Verstärkt wird dies durch die Tatsache. dass diese Krankheit in unserer Gesellschaft weitgehend tabuisierte Themen wie Tod, Sexualität und Rausch bzw Sucht berührt. Es ist eine Krankheit, in deren sprachlichem Umfeld häufig in kriegsähnlichen Bildern aufgerüstet und gekämpft wird. Da ein Grossteil der durch AIDS Betroffenen gesellschaftlichen Randgruppen angehört, befindet sich diese «Mobilmachung» in Gefahr, ihr Ziel - die Krankheit - zu verfehlen und stattdessen die Kranken zu treffen, zB: in Form stigmatisierender, ausgrenzender Massnahmen.

Die soziale Gestalt einer Krankheit wird von Menschen gemacht und kann von Menschen wieder verändert werden. Dies gilt auch für AIDS. Hier liegt neben der medizinischen die zentrale soziale Herausforderung durch AIDS. Vergessen wir deshalb nicht: Wir bestimmen heute durch unser Denken und Handeln das Bild, unter dem AIDS in die Geschichte der Krankheiten und des sozialen Umgangs mit ihnen eingehen wird: Als eine Krankheit der Diskriminierung und Ausgrenzung oder als eine Krankheit der mitmenschlichen Anteilnahme und Solidarität.

Rainer Hornung, Felix Gutzwiller; Zürich

[1] Gorsboth $T$, Wagner B. Die Unmöglichkeit der Therapie. Kursbuch «Die Seuche» (94). Berlin: Kursbuch Verlag, 1988: $123-164$.

[2] Sontag S. Krankheit als Metapher. München: Hanser, 1978.

[3] Sontag S. AlDS und seine Metaphern. München: Hanser. 1989. 


\section{Editorial}

\section{De l'image sociale des maladies}

La tuberculose et le SIDA sont caractéristiques chacun d'une époque particulière de la longue histoire des maladies transmissibles. Cette année, lors des Journées scientifiques de la Société Suisse de Médecine sociale et préventive, une série d'exposés leur ont été consacrés: «Tuberkulose bei Schweizern und Ausländern: Präventivmedizinische Implikationen», «Epidemiologische Analyse des Grenzsanitätsdienstes», "Evaluation du dépistage de la tuberculose dans une population d'apprentis et de gymnasiens vaudois", «Effektorientierte Evaluation der AIDS-Aufklärung an Zürcher Volksschulen», «Préserver ses chances matrimoniales sans s'exposer au risque du SIDA - le cas des femmes divorcées». La session de clôture avait pour thème: «Discrimination et stigmatisation des personnes atteintes du SIDA».

La tuberculose est l'exemple d'une maladie que l'on croit à tort rattachée au passé; il suffit de penser à l'impressionnante recrudescence de cas mortels de tuberculose à New-York entre 1979 et 1986 [1]; les principales victimes furent les sans-abri et les toxicomanes. la maladie reflétant les inégalités sociales.

De manière dramatique, le SIDA vient à son tour démentir de manière dramatique la croyance que l'ère des maladies transmissibles est définitivement révolue pour les sociétés développées. Cette maladie, dont les média ne cessent de parler, suscite de nombreux débats de société et de politique sociale.

Par ailleurs, toute maladie possède une «image " sociale faite de toutes les représentations que lui attribue l'imagination collective, ainsi que le montre Susan Sontag [2] dans un brillant essai intitulé «La maladie comme métaphore». Par exemple, le cancer est souvent synonyme de mort; c'est une maladie «démoniaque» qui symbolise le mal et le mot «cancer» lui-même est employé dans ce sens dans le langage de tous les jours. Par contraste, la tuberculose est entourée d'une aura sacrée et romantique: c'est la maladie des âmes hypersensibles, dominées par la passion. L'illustration la plus significative en est peut-être donnée par le célèbre roman de Thomas Mann «La Montagne Magique».

Qu'en est-il du SIDA? Dans un ouvrage récent [3], Susan Sontag s'est intéressée aux métaphores qui s'attachent à cette maladie du système immunitaire. Apparu en cette fin du vingtième siècle et touchant aux domaines tabous de la sexualité, de la jouissance et de la mort, le SIDA permet d'exprimer toutes les peurs apocalyptiques, et toutes les projections catastrophistes. Une telle maladie doit être conjurée à tolit prix; des stratégies, des campagnes et des plans de bataille sont élaborés. Cependant, comme la majorité des personnes atteintes du SIDA vivent en marge de la société, une entrée en guerre accompagnée de mesures discriminatoires ou stigmatisantes risque de ne pas atteindre son objectif, qui est de circonscrire la maladie, et d'isoler encore davantage les malades.

Si les hommes peuvent charger une maladie de valeurs positives ou négatives, celle-ci, en retour, va véhiculer dans l'histoire une certaine image de son contexte social. Et cette image-là, il ne faut pas l'oublier, ce sont nous-mêmes qui sommes en train de la déterminer par nos façons de penser et nos actions. A côté du défi médical, il s'agit là de la question essentiel le que nous pose l'apparition du SIDA: notre société est-elle basée sur la discrimination et l'exclusion ou, au contraire, est-elle fondée sur la convivialité et la solidarité humaine?

Rainer Hornung, Felix Gutzwiller; Zurich

[1] Gorsboth T. Wagner B. Die Unmöglichkeit der Therapie. Kursbuch "Die Seuche $(94)$. Berlin: Kursbuch Verlag. 1988: 123-164.

[2] Sontag S. Krankheit als Metapher. München: Hanser, 1978.

[3] Sontag S. AIDS und seine Metaphern. München: Hanser, 1989. 